\title{
FABRICATION AND TENSILE PROPERTY ANALYSIS OF A COMPOSITE LAMINATE WITH DIFFERENT DIAMETER HOLES
}

\author{
M.Ravindra Gandhi ${ }^{1}$, P.V.R.Girish Kumar ${ }^{2}$, P.Ashok Reddy ${ }^{3}$, B.Siva Prasad $^{4}$ \\ ${ }^{1}$ Assistant professor, Mechanical Engineering, Geethanjali college of engineering and Technology, Telangana, India \\ ${ }^{2}$ Assistant professor, Mechanical Engineering, Geethanjali college of engineering and Technology, Telangana, India \\ ${ }^{3}$ Assistant Professor, Mechanical Engineering, Vignan Institute of Technology and science, Telangana, India \\ ${ }^{4}$ Assistant Professor, Mechancial Engineering, Vignan Institute of Technology and science, Telangana, India
}

\begin{abstract}
Composites play a vital role in aerospace, land transportation and consumer goods due to their high specific strength and stiffness leading to reduction in the mass of moving objects. Some of the important aerospace hardware such as rocket motor casing makes extensive use of carbon fiber reinforced polymer(CFRP). In this present investigation E-Glass epoxy laminates were used. The tensile properties for various Diameter holes were determined and compared with the actual strength of the material without any hole. Results indicate that because of the increase in diameter of the hole the tensile strength of the material is decreasing.
\end{abstract}

Keywords: CFRP, E-Glass, Epoxy, Tensile strength.

\section{INTRODUCTION}

In the field of mechanical \& aerospace composites are widely used because of the high specific strength and low density of composites. Composites are widely used because of their tailorability of properties to the required direction or strength. In the field of aerospace, for the sake of pressure vessels like rocket motor casings composites will result in high strength as well as low weight. Weight is a major factor of any flying object whenever we discuss about trade off ratios. So for the sake of reduced weight and increased FOS composites are emerging materials now days.

Mechanical performance of fibrous composites will depends on the following aspects

1. Surface treatment of the fiber

2. Percemtage elongation of the fiber \& matrix

3. Chemistry of the resin dictated by functionality, addition of toughening agent, curing agents and curing conditions.

\section{LAMINATE PREPARATION}

E-glass/epoxy resin laminates were prepared by drum winding process. E-glass fibres are passed through a resin bath containing epoxy resin mixed with $10 \%$ by weight of hardener HY 5200.The traction for pulling resin wetted fibres which pass through a pay-out eye attached to the longitudinal slide (tool post), is provided by rotating cylindrical mandrel. The resin bath is equipped with in-built servo controlled heating system and the temperature of resin mix is maintained at $40^{\circ} \mathrm{c}$, in order to bring the resin viscosity to around 1000-1500cpa.This enables the volume fraction of fibres to be at around $60 \%$. The cylindrical drum on which fibres are deposited is rotated at a low speed of $15 \mathrm{rpm}$. Prior to winding, it is covered with a thick HDPE film without any wrinkles what so ever. After the winding is completed, the mandrel kept under rotation for about an hour to ensure that there is no resin collection at the bottom due to gravity. The punch plate is then assemble to it and clamped with bolts \& nuts. The clamping bolts are torqued to a value of $30 \mathrm{~N}-\mathrm{m}$ with the help of a torque wrench. The assembly is placed in a curing oven and the composite was cured. as per the following general cure cycle.

Variations were made to suit the resin systems as recommended by the manufacturer.

Room temperature to $70 \mathrm{oC}$ - 3omin

Hold at $70 \mathrm{oC}-120 \mathrm{~min}$

Ramp to $150 \mathrm{oC}-30 \mathrm{~min}$

Hold at $150 \mathrm{oC}-240 \mathrm{~min}$

Thereafter remove the composite laminate from mouldand trim the edges.

To prepare the laminate, the following process parameters were used

\begin{tabular}{|l|l|l|}
\hline SNo & Parameter/Material & Details \\
\hline 1 & Resin/Hardener & LY-556/HY 5200 \\
\hline 3 & Tool & $\begin{array}{l}\text { Cylindrical/Rectangular } \\
\text { Mandrel }\end{array}$ \\
\hline 4 & Process & a)Drum winding \\
\hline 5 & Band width & $3.0 \mathrm{~mm}$ \\
\hline 6 & Fibre Tension & $0.9 \mathrm{~kg}$ \\
\hline 7 & $\begin{array}{l}\text { Distance between } \\
\text { Resin bath and } \\
\text { mandrel }\end{array}$ & \begin{tabular}{l}
$1.66 \mathrm{~m}$ \\
\hline 8
\end{tabular} \\
\hline
\end{tabular}




\begin{tabular}{|l|l|l|}
\hline 9 & Mandrel RPM & $15 \mathrm{RPM}$ \\
\hline 10 & Resin temperature & $45^{\circ} \mathrm{C}$ \\
\hline 11 & Volume fraction & $60 \%$ \\
\hline
\end{tabular}

Specimen Preparation: Specimens were prepared from composite laminated plates according to ASTM specifications

ASTM Specifications: Specimens were prepared from composite laminated plates; the manufacturing process is described under the heading "material preparation". The specimens conform to the requirements lead down in the relevant ASTM specifications listed below

\begin{tabular}{|l|l|l|l|l|}
\hline S.No & $\begin{array}{l}\text { Type of } \\
\text { test }\end{array}$ & $\begin{array}{l}\text { Relevant } \\
\text { ASTM }\end{array}$ & $\begin{array}{l}\text { Number } \\
\text { of } \\
\text { specimens }\end{array}$ & $\begin{array}{l}\text { Specimen } \\
\text { size(mm) }\end{array}$ \\
\hline 1 & $\begin{array}{l}\text { UD- } \\
\text { Tensile }\end{array}$ & D-3039 & 5 & $250 \times 15 \times 1.0$ \\
\hline 2 & $\begin{array}{l}\text { Transverse } \\
\text { tensile }\end{array}$ & D-3039 & 5 & $175 \times 25 \times 2.0$ \\
\hline 3 & Flexural & D-790 & 5 & $50 \times 25 \times 1.6$ \\
\hline 4 & ILSS & D-2344 & 5 & $24 \times 12 \times 6.0$ \\
\hline 5 & $\begin{array}{l}\text { In plane } \\
\text { shear }\end{array}$ & D-3518 & 5 & $300 \times 25 \times 3.0$ \\
\hline
\end{tabular}

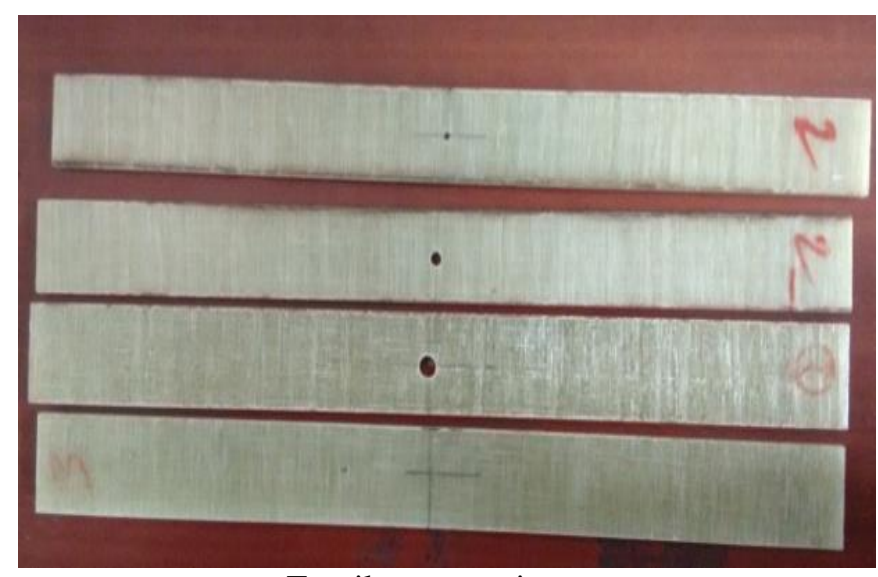

Tensile test specimen

Tensile Properties without a hole

\begin{tabular}{|l|l|l|l|}
\hline S.No & $\begin{array}{l}\text { Geometry } \\
(\mathbf{m m})\end{array}$ & BreakingLoad(KN) & $\boldsymbol{\sigma ( \mathrm { MPa } )}$ \\
\hline 1. & $\begin{array}{l}300 \times 27.27 \times \\
3.8\end{array}$ & 59.6 & 575.14 \\
\hline 2. & $\begin{array}{l}300 \times 27.8 \times \\
3.9\end{array}$ & 58.5 & 539.56 \\
\hline 3. & $\begin{array}{l}300 \times 27.8 \times \\
5.1\end{array}$ & 80.5 & 567.78 \\
\hline
\end{tabular}

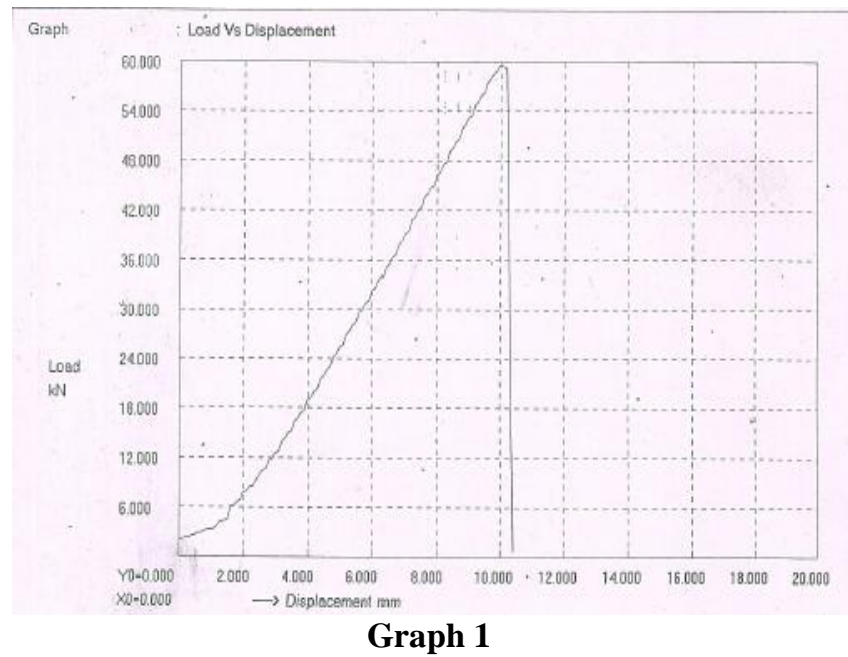

Tensile Properties with 3mm centre hole:

\begin{tabular}{|l|l|l|l|}
\hline S.No & $\begin{array}{l}\text { Geometry } \\
(\mathbf{m m})\end{array}$ & BreakingLoad(KN) & $\sigma(\mathbf{M P a})$ \\
\hline 1. & $\begin{array}{l}300 \times 26.5 \times \\
3.8\end{array}$ & 40.5 & 402.18 \\
\hline 2. & $\begin{array}{l}300 \times 28.5 \times \\
4.0\end{array}$ & 47.0 & 412.28 \\
\hline 3. & $\begin{array}{l}300 \times 26.6 \times \\
5.15\end{array}$ & 61.46 & 448.64 \\
\hline
\end{tabular}

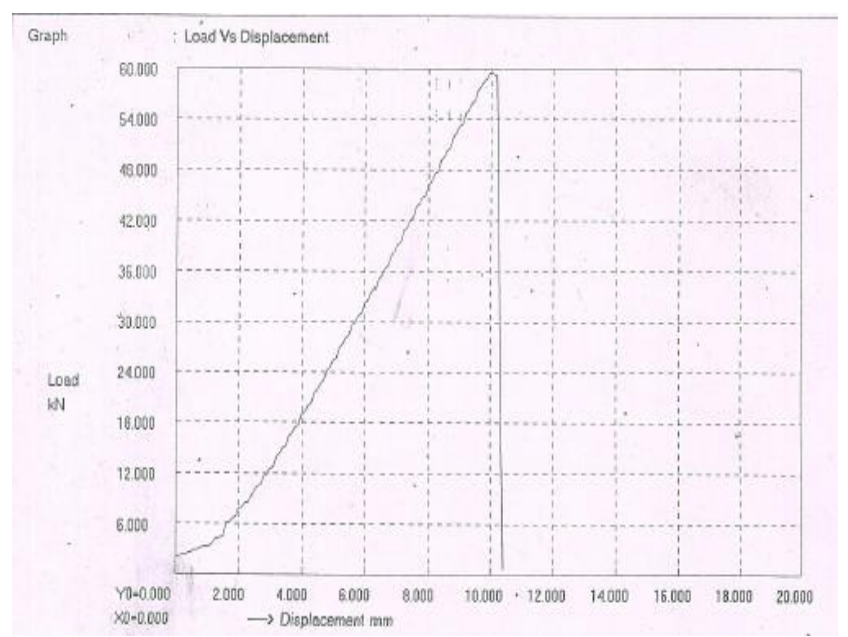

Graph 2

Tensile Properties with 3mm offset hole

\begin{tabular}{|l|l|l|l|}
\hline S.No & $\begin{array}{l}\text { Geometry } \\
(\mathbf{m m})\end{array}$ & BreakingLoad(KN) & $\sigma(\mathrm{MPa})$ \\
\hline 1. & $\begin{array}{l}300 \times 26.4 \times \\
3.8\end{array}$ & 35.0 & 348.88 \\
\hline 2. & $\begin{array}{l}300 \times 28.0 \times \\
4.0\end{array}$ & 46.0 & 410.71 \\
\hline 3. & $\begin{array}{l}300 \times 26.0 \times \\
5.1\end{array}$ & 61.4 & 463.04 \\
\hline
\end{tabular}




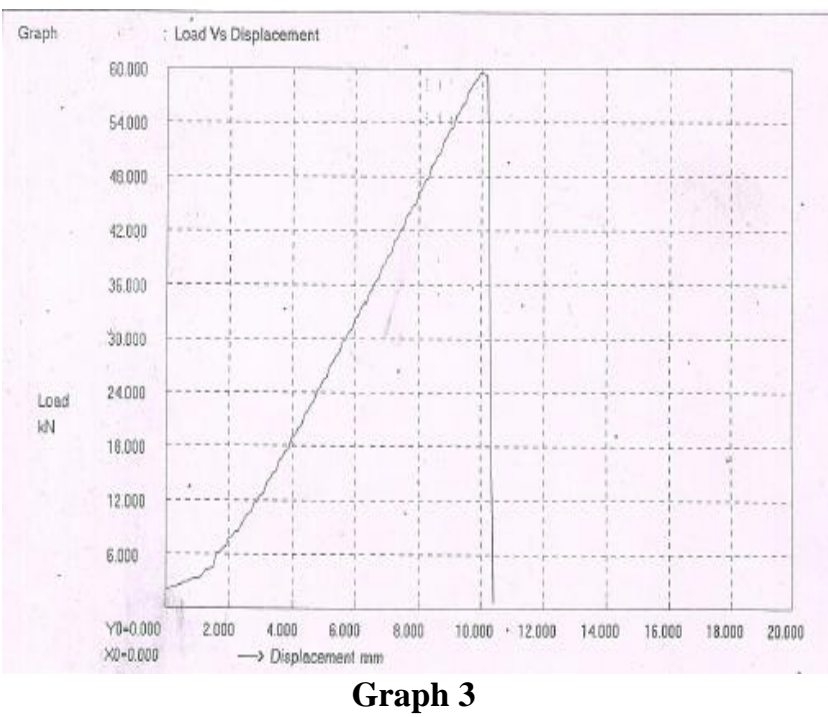

Tensile Properties with 5mm centrehole:

\begin{tabular}{|l|l|l|l|}
\hline S.No & $\begin{array}{l}\text { Geometry } \\
(\mathbf{m m})\end{array}$ & BreakingLoad(KN) & $\sigma(\mathrm{MPa})$ \\
\hline 1. & $\begin{array}{l}300 \times 26.0 \times \\
3.68\end{array}$ & 34.5 & 360.57 \\
\hline 2. & $\begin{array}{l}300 \times 28.0 \times \\
3.85\end{array}$ & 39.5 & 366.42 \\
\hline 3. & $\begin{array}{l}300 \times 26.75 \times \\
5.15\end{array}$ & 58.92 & 427.69 \\
\hline
\end{tabular}

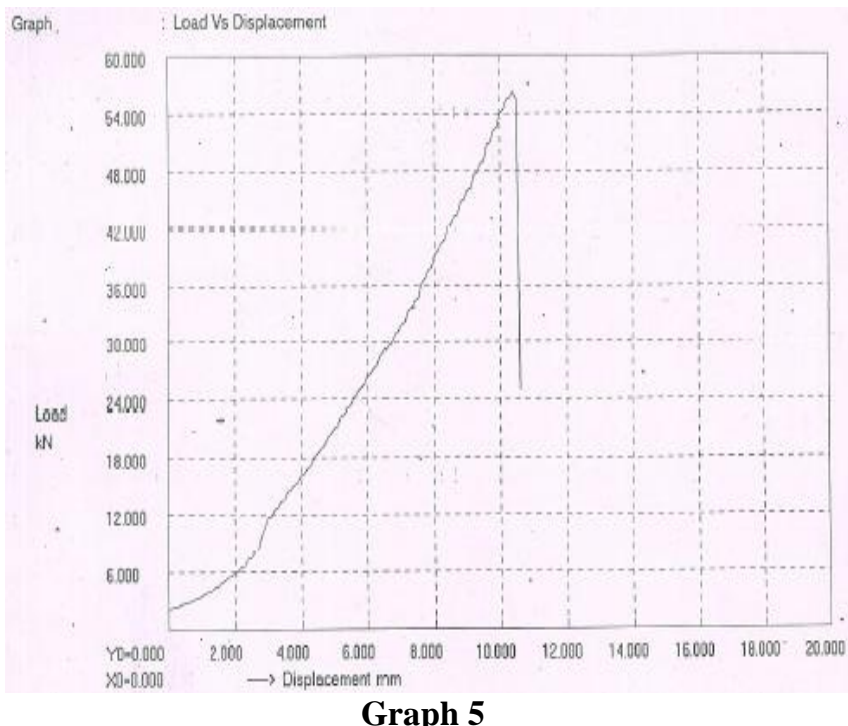

Tensile Properties with $7 \mathrm{~mm}$ centre hole:

\begin{tabular}{|c|c|c|c|}
\hline S.No & $\begin{array}{l}\text { Geometry } \\
(\mathrm{mm})\end{array}$ & BreakingLoad(KN) & $\sigma(\mathrm{MPa})$ \\
\hline 1. & $\begin{array}{l}300 \times 27.0 \times \\
3.8\end{array}$ & 32.5 & 316.76 \\
\hline 2. & $\begin{array}{llll}300 & \mathrm{x} & 27.8 & \mathrm{x} \\
4.0 & & & \\
\end{array}$ & 33.5 & 301.26 \\
\hline 3. & $\begin{array}{l}300 \times 26.2 \times \\
5.1\end{array}$ & 47.22 & 353.39 \\
\hline
\end{tabular}

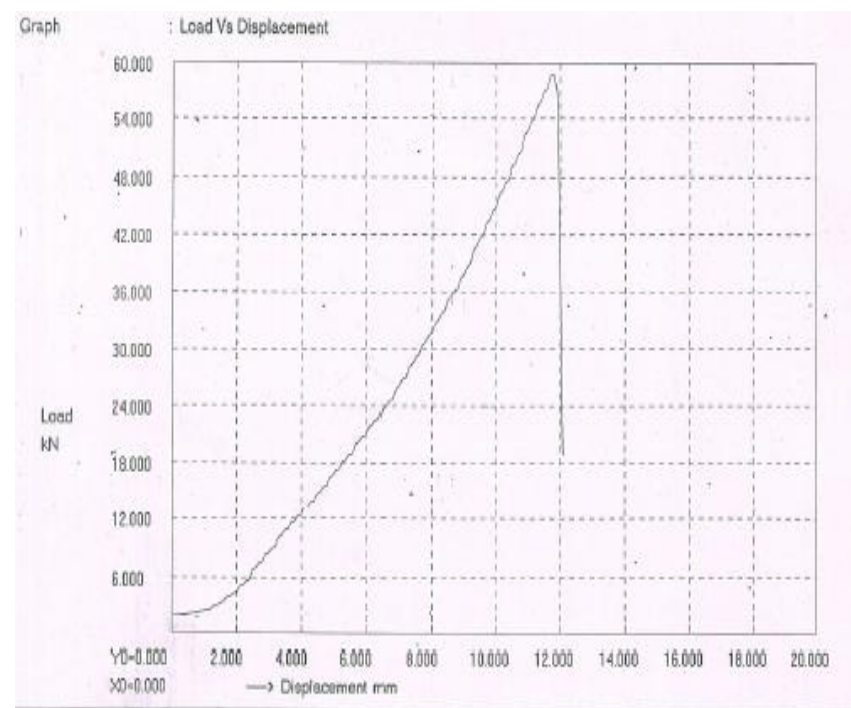

\section{Graph 4}

Tensile Properties with 5mm offset hole:

\begin{tabular}{|l|l|l|l|}
\hline S.No & $\begin{array}{l}\text { Geometry } \\
(\mathbf{m m})\end{array}$ & BreakingLoad(KN) & ((MPa) \\
\hline 1. & $\begin{array}{l}300 \times 26.25 \times \\
3.8\end{array}$ & 33.5 & 335.84 \\
\hline 2. & $\begin{array}{l}300 \times 27.32 \times \\
3.8\end{array}$ & 34.0 & 327.5 \\
\hline 3. & $\begin{array}{l}300 \times 26.75 \times \\
5.1\end{array}$ & 56.24 & 412.24 \\
\hline
\end{tabular}

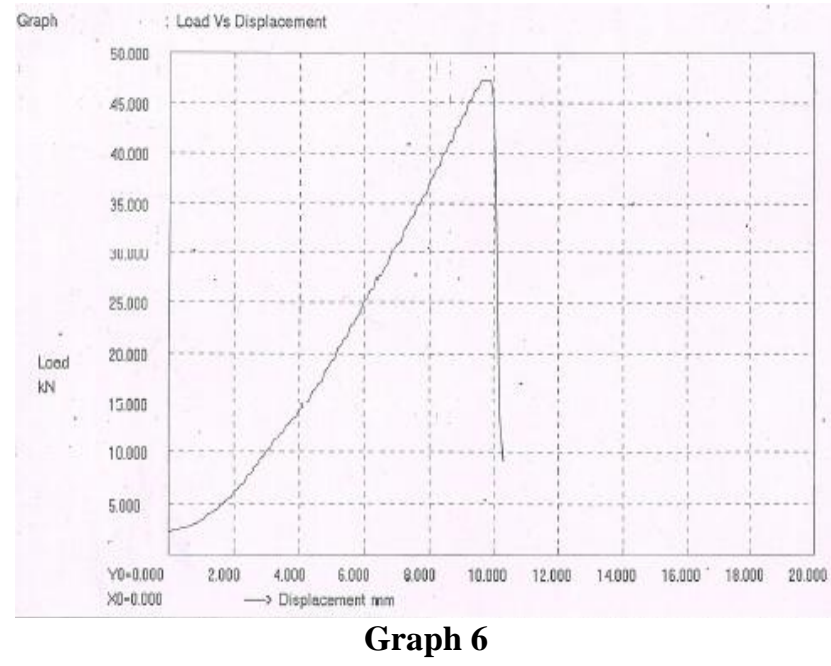

Tensile Properties with $7 \mathrm{~mm}$ offset hole:

\begin{tabular}{|l|l|l|l|}
\hline S.No & $\begin{array}{l}\text { Geometry } \\
(\mathbf{m m})\end{array}$ & BreakingLoad(KN) & (MPa) \\
\hline 1. & $\begin{array}{l}300 \times 26.3 \times \\
3.85\end{array}$ & 29.4 & 290.35 \\
\hline 2. & $\begin{array}{l}300 \times 27.0 \times \\
4.0\end{array}$ & 30.82 & 285.37 \\
\hline 3. & $\begin{array}{l}300 \times 27.2 \times \\
5.1\end{array}$ & 46.7 & 336.65 \\
\hline
\end{tabular}




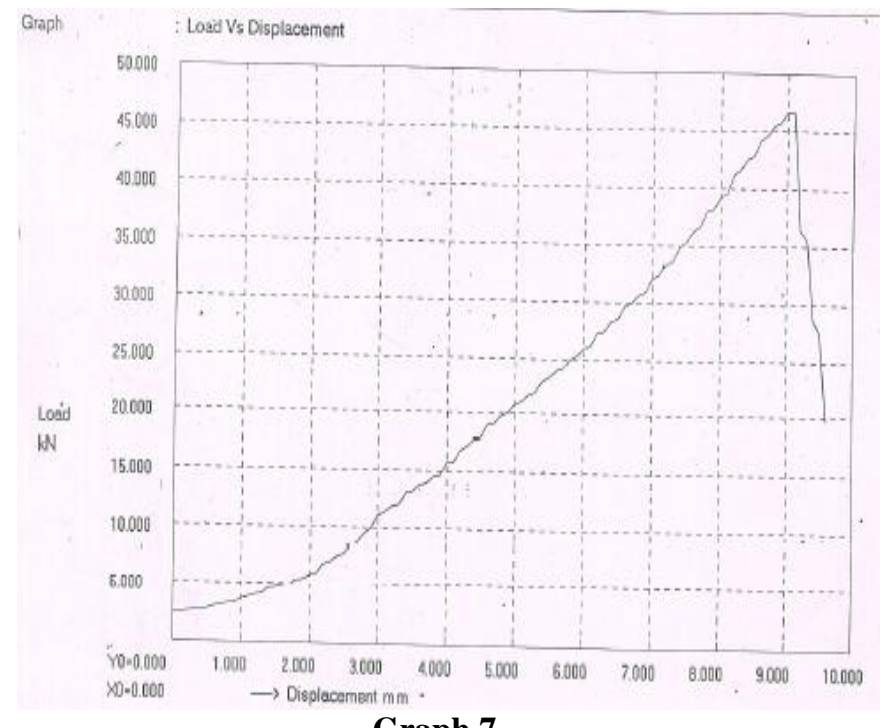

Graph 7

\section{RESULTS \& CONCLUSION}

1. Tensile strength of samples without hole $=560.82 \mathrm{MPa}$

2. Tensile strength of samples with $3 \mathrm{~mm}$ hole at center $=421.03 \mathrm{MPa}$

3. Tensile strength of samples with $3 \mathrm{~mm}$ hole at $5 \mathrm{~mm}$ offset from the center $=407.54 \mathrm{MPa}$

4. Average Tensile strength of samples with $5 \mathrm{~mm}$ hole at center $=384.89 \mathrm{MPa}$

5. Average Tensile strength of samples with $5 \mathrm{~mm}$ hole at $5 \mathrm{~mm}$ offset from the center $=358.52 \mathrm{MPa}$

6. Average Tensile strength of samples with $7 \mathrm{~mm}$ hole at center $=323.80 \mathrm{MPa}$.

The Tensile strength of the specimen is inversely proportional to the Diameter of the hole.The Tensile strength is lower for specimen having hole at offset from the centre compared to specimen with hole at the centre.

\section{REFERENCES}

[1] Topdar, A. H. Sheikh and N. Dhang Finite Element Analysis of Composite and Sandwich Plates Using a Continuous Inter-laminar Shear Stress Model P. Journal of Sandwich Structures and Materials 2003; 5; 207.

[2] Robert Matteson and Roger Crane, "Flexural testing of steel wire composite beams made with Hardwire unidirectional tape,'NSWCCD-65-TR-2003/48 Nov. 2003 (NASA Technical Report).

[3] P.D.Bradley, S.J. Harris, Strategic reinforcement of hybrid carbon fibers reinforced polymer composites, J. Mater. Sci.12 (1977), pp. 2401-2410

[4] Dr. Jawad Kadhim Uleiwi, Experimental Study of Flexural Strength of Laminate Composite Material, Eng. \& Technology, Vol.25, Suppl.of No.3, 2007, pp 454-466.

[5] Slimane Metiche and Radhouane Masmoudi, FullScale Flexural Testing on Fiber-Reinforced Polymer (FRP) Poles, The Open Civil Engineering Journal, , 1, 37-50, 2007, pp 37-50.
[6] M. Davallo, H. Pasdar and M. Mohseni, Effects of Laminate Thickness and PlyStacking Sequence on the Mechanical Properties and Failure Mechanism of Unidirectional Glass-Polyester Composites, International Journal of ChemTech 41 Research, CODEN( USA): IJCRGG ISSN : 0974-4290, Vol.2, No.4, Oct-Dec 2010, pp 2118-2124.

[7] Michel Espinosa Klymus, Rosemary Sadami Arai Shinkai, Eduardo Gonealves Mota, Influence of the mechanical properties of composites for indirect dental restorations on pattern failure,Stomatologija, Baltic Dental and Maxillofacial Journal, Vol. 9, 2007, pp. 56-60.

[8] S.Benjamin Lazarus, V. Vel Murugan, Experimental Investigation for Mechanical Properties of Chopped Random Fibre Compression Moulded Sunnhemp Polyester Composites, European Journal of Scientific Research, ISSN 1450-216X Vol.82 No.3, 2012, pp.366-380.

[9] I. J. Davies and H. Hamada, Flexural properties of a hybrid polymer matrix composite containing carbon and silicon carbide fibres, Adv. Composite Maters., 10(1), 2001, pp. 77-96.

[10] Hoo Tien Kuan, Wesley Cantwell and Hazizan Md Akil, The Mechanical Properties of Hybrid Composites Based on Self-Reinforced Polypropylene, Malaysian Polymer Journal, Vol. 4, No.2, 2009, p 71-80

[11] N.K. Naik, Addis Asmelash, Venkateswara Rao Kavala, Veerraju Ch, Interlaminar shear properties of polymer matrix composites: Strain rate effect.

\section{BIOGRAPHIES}

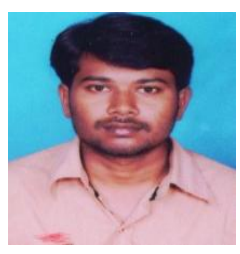

M.Ravindra Gandhi, Assistant professor, Mechanical Engineering, Geethanjali college of engineering and Technology, Telangan, India

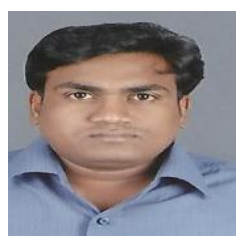

P.V.R.Girish Kumar, Assistant professor, Mechanical Engineering, Geethanjali college of engineering and Technology, Telangan, India

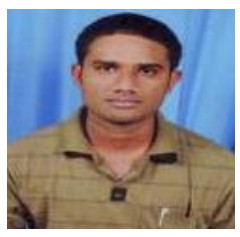

P.Ashok Reddy, Assistant professor, Mechanical Engineering, Vignan Institute of Techonlogy \& Science, Telangan, India

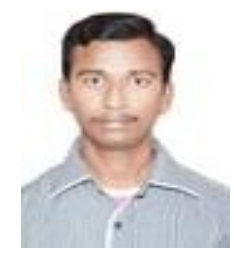

B.Siva Prasad, Assistant professor, Mechanical Engineering, Vignan Institute of Techonlogy \& Science, Telangan, India 